\title{
A Study on the Stable Sensorless Control of BLDC Motor Inside Auxiliary Air Compressor
}

\author{
In-Gun Kim*, Hyun-Seok Hong*, Sung-Chul Go**, Ye-Jun Oh*, Kyoung-Jin Joo* \\ and $\mathrm{Ju} \mathrm{Lee}{ }^{\dagger}$
}

\begin{abstract}
Pantograph must be correctly attached to catenary to continuously supply stable power to railway vehicle, and the device used here is Auxiliary Air Compressor (ACM). The existing ACM used the DC motor that included commutator and brush. Since maintenance and repair by mechanical friction are essential for the DC motor, BLDC motor studies have been conducted to improve this. A three-phase BLDC motor does $120^{\circ}$ two-phase commutation through hall sensors in general. However, since hall sensor is vulnerable to heat and can run only when all three sensors work normally, sensorless control method has been studied to solve this. Using back EMF Zero Crossing Point (ZCP) detection method, this paper will introduce a stable switching sensing method that has a noncommutation area in a low speed zone.
\end{abstract}

Keywords: Auxiliary air compressor, Non-commutation area, BLDC motor, Sensorless control, Zero crossing point(ZCP), Pure back EMF

\section{Introduction}

Auxiliary Air Compressor (ACM) is used to continuously supply stable alternating current power to railway vehicle facilities [1]. ACM increases pantograph toward catenary to be correctly attached when maneuvering a railway vehicle [2]. The compressed air of ACM is generated by rotating the motor and must be controlled so that the actual discharge rate is maintained consistently compared to the discharge pressure. The existing ACM has used DC motor that does not inverter but requires mechanical driving to secure reliability. DC motor is excellent in terms of its functions but has brush and commutator, so friction must always be considered when it is driving and maintenance is essential as abrasion increases. Improper maintenance disables continuous rotation to a consistent direction and cannot satisfy the rated torque. Thus, an alternative study is currently ongoing on Brushless DC motor (BLDCM) which enables electrical communication and does not need to consider mechanical friction during commutation [3-5].

Since BLDC motor can be developed in a three-phase circuit, it can improve power density more than DC motor in the same size, enables PWM control as it uses threephase inverters, and can control the voltage duty ratio. However, unlike DC motor, it needs additional three-phase

$\dagger$ Corresponding Author: Dept. of Electrical Engineering, Hanyang University, Korea. (julee@hanyang.ac.kr)

* Dept. of Electrical Engineering, Hanyang University, Korea (waterkig@naver.com, hhs0321@gmail.com, oyejuni@naver.com, kj8532@hanyang.ac.kr)

** Dept. of Mechatronics R\&D Center. Mechatronics Group. Samsung Electronics Co., Ltd., Korea. (sc.go@samsung.com)

Received: July 6, 2016; Accepted: September 28, 2016 inverters and requires a position sensor for accurate commutation. Generally, BLDC motor is appropriate for applications that do not have frequent speed variability due to the nature of its use conditions, and does not need to drive by measuring the absolute position of rotor. Therefore, it is cheaper than a resolver or encoder and uses a position sensor of hall type that enables driving as long as the polarity position of rotor is distinguished. Due to its cheap price and relatively simple structure, hall sensor is often used for BLDC motor control. However, since it is also vulnerable to heat and identifies the position only by polarity, it can result in switch sensing errors and inverter damage even when an error occurs in only one of the three sensors [6-8].

To solve this problem, much study has been conducted on sensorless control. The sensorless method that is relatively simple and is widely used is the back EMF detection method, which detects the ZCP of back EMF, is a method used for commutation, and can simply develop an external circuit [9-11]. However, compared to the sensor type, its weakness is that it has decreasing

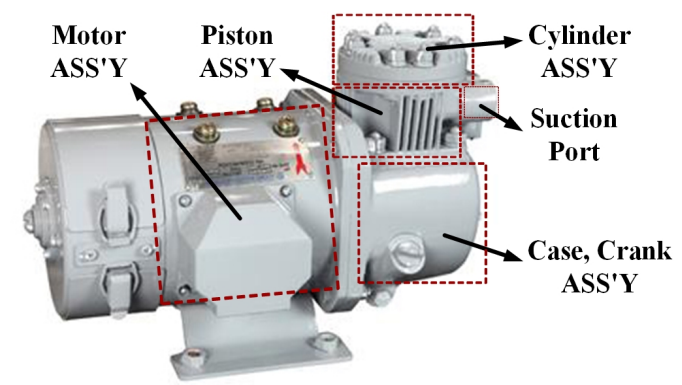

Fig. 1. ACM applied on urban railway vehicle 
reliability of rotor position because it cannot secure the position accuracy of transient state zone, where start-up and speed variability occur. To overcome the problem of the existing sensorless control method, this study proposes a ZCP detection method without noise by setting a noncommutation area during medium speed driving. This method enables a stable sensorless control until reaching a rated speed without additional circuit formation. Fig. 1 shows an ACM structure using the existing DC motor.

\section{Sensorless Control of BLDC Motor}

\subsection{Design of BLDC motor to substitute DC motor inside $\mathrm{ACM}$}

The existing ACM (MH99-AK19) is currently installed and used in lines no. 9 of Seoul subway, and it generates compressed air by maintaining consistent discharge pressure through DC motor. DC motor has a brush and commutator in general, so it is commutated when the two structures come into contact and can rotate to a consistent direction; however, friction occurs due to its structure and thus requires maintenance as abrasion increases accordingly. On the contrary, BLDC can be commutated electrically as it uses an inverter structure, so mechanical friction does not need to be considered like DC motor. Table 1 shows the specifications of the existing DC motor and newly designed BLDC motor.

Based on the rated specifications of designed DC motor, the design was carried out to obtain the same power and torque from the BLDC motor. The motor was modeled through the finite elements method, the number of poles was the same as 4 poles of the existing DC motor, and the

Table 1. Specifications of DC motor and BLDC

Designed BLDC Motor Specification

\begin{tabular}{|c|c|c|c|}
\hline \multicolumn{2}{|l|}{ Parameter } & Value & Unit \\
\hline \multicolumn{2}{|l|}{ Motor Type } & IPMSM & - \\
\hline \multicolumn{2}{|l|}{ Rated Power } & 400 & $\mathrm{~W}$ \\
\hline \multicolumn{2}{|l|}{ Pole / Slot } & $4 / 6$ & - \\
\hline \multicolumn{2}{|l|}{ Rated Speed } & 3000 & $\mathrm{rpm}$ \\
\hline \multicolumn{2}{|l|}{ Core Type } & 20PNF1500 & - \\
\hline \multirow{2}{*}{ Permanent Magnet } & $B_{r}$ & 1.18 & $\mathrm{~T}$ \\
\hline & $\mu_{r}$ & 1.06 & - \\
\hline \multicolumn{2}{|c|}{ DC Input Voltage } & 80 & $\mathrm{~V}$ \\
\hline \multicolumn{2}{|c|}{ Phase Current } & 4.81 & Arms \\
\hline \multicolumn{2}{|l|}{ Rated Torque } & 2.35 & $\mathrm{Nm}$ \\
\hline
\end{tabular}

\begin{tabular}{c|c|c}
\multicolumn{3}{c}{ DC Motor Specification } \\
\hline Parameter & Value & Unit \\
\hline Motor Type & DC Motor & - \\
\hline Rated Power & 400 & $\mathrm{~W}$ \\
\hline Rated Speed & 3000 & $\mathrm{rpm}$ \\
\hline DC Input Voltage & 80 & $\mathrm{~V}$ \\
\hline Phase Current & 5.1 & Arms \\
\hline Rated Torque & 2.2 & $\mathrm{Nm}$ \\
\hline
\end{tabular}

number of slots were set at 4 poles and 6 slots for the combination $(2 / 3)$ of the number of poles and number of slots considering its making. Since heat is generated inside the motor due to the copper loss of stator winding, it is more favorable to protect against the heat if more surface area of slots inside is secured inside. Therefore, the tooth concentrated winding method was applied to secure the surface area of slots as much as possible by increasing the coil space factor. Fig. 2 is the model and external linkage circuit for finite elements method of BLDC motor that replaces DC motor. Fig. 3 is the $120^{\circ}$ two-phase commutation method showing the motor phase current and torque waveform in which finite elements method was applied [12].
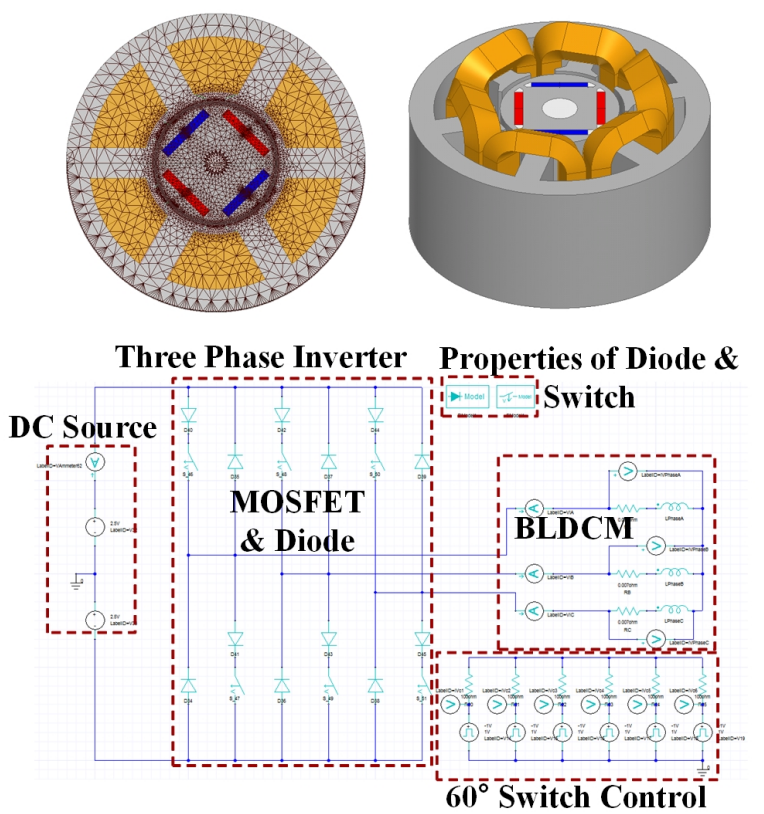

Fig. 2. Modeling and external linkage circuit diagram of BLDC motor designed

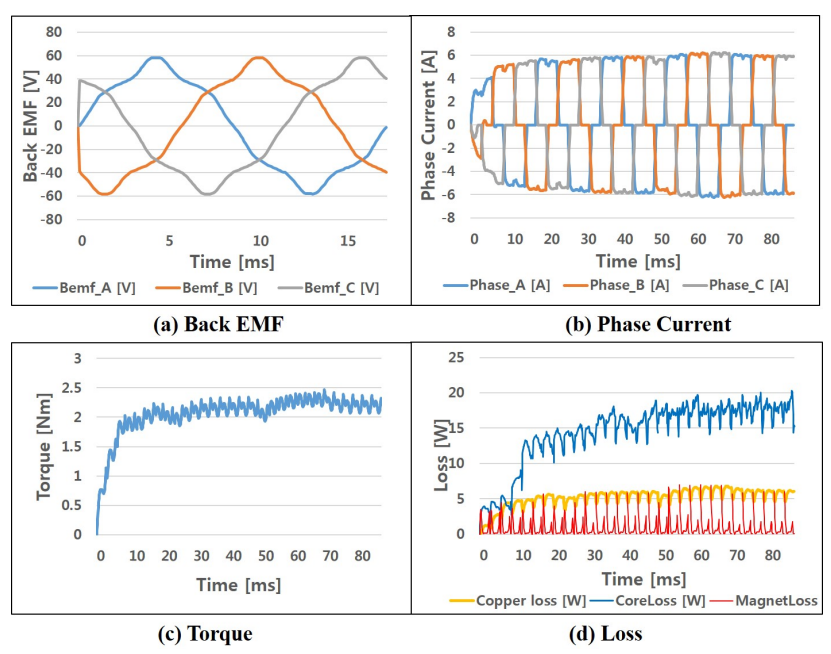

Fig. 3. Back EMF, Phase current, torque waveform and total loss of BLDC motor designed 


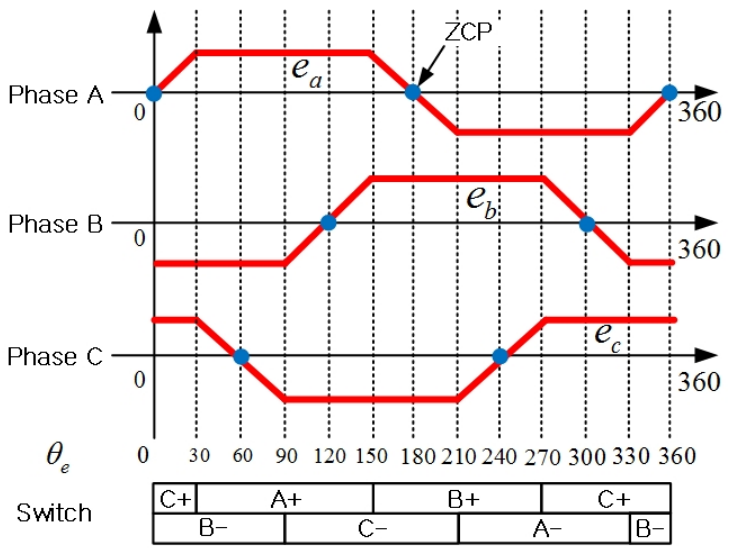

Fig. 4. Back EMF, ZCP, electrical angle, and switching sequence of BLDC motor

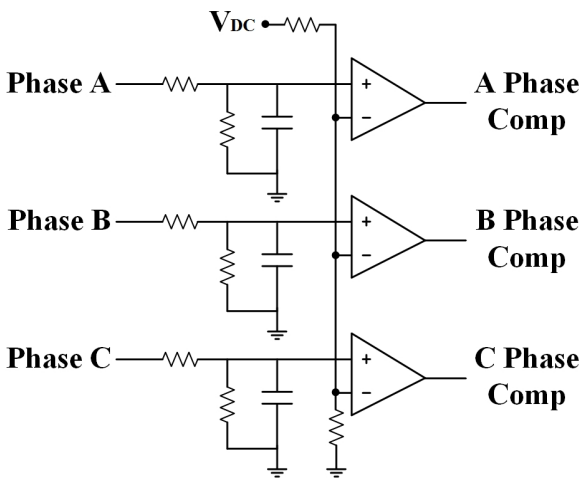

Fig. 5. ZCP detection circuit using comparator three

\subsection{ZCP detection using back EMF}

The sensorless control method of BLDC motor often uses a method that detects the ZCP of back EMF included in terminal voltage. This is because it enables control by forming a relatively simple external circuit and achieves the maximum size of back EMF once the motor reaches the rated speed, making ZCP detection easier. Fig. 4 shows back EMF, ZCP(blue point), electrical angle, and switching sequence.

The back EMF in Fig. 4 is an ideal waveform, and it is developed in PWM pulse form as it included in terminal voltage as in Formula 1 during the actual motor driving. $V_{s}, V_{a}, V_{n}, e_{a}$ are terminal voltage, phase voltage, neutral voltage and back EMF.

$$
V_{s}=V_{a}+V_{n}=R_{a} i_{a}+L_{a} \frac{d i_{a}}{d t}+e_{a}+V_{n}
$$

The external circuit for ZCP detection uses OP-AMP and resistance, capacitor, and buffer. Each of OP-AMP is used for comparator and resistance, capacitor for low pass filter, and buffer for maintaining the voltage level. The signal of each phase can be detected using three comparators as in Fig. 5 [13], but ZCP can be detected by one comparator

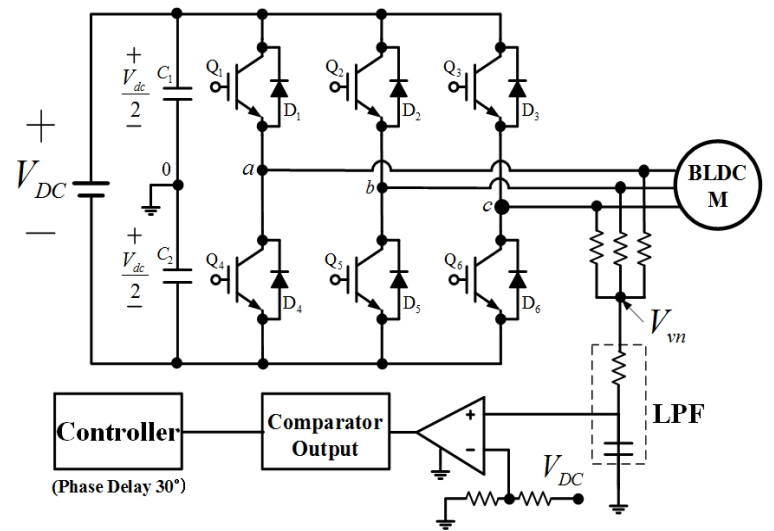

Fig. 6. ZCP detection circuit using one proposed comparator

when using virtual neutral point voltage. Virtual neutral point can be expressed by the sum of the terminal voltage of three-phase and is shown in a triangular wave form. Then, ZCP is found by comparing this waveform with $\mathrm{Vdc} / 2$. Virtual neutral point can be expressed as in Formula 2 , and Fig. 6 is the proposed ZCP detection circuit. $V_{v n}$ is virtual neutral point voltage. $V_{s a}, V_{s b}, V_{s c}$ are terminal voltage of phase A to $\mathrm{C}$.

$$
V_{v n}=\frac{V_{s a}+V_{s b}+V_{s c}}{3}
$$

\subsection{Driving method depending on speed zone}

BLDC sensorless control method must be considered by classifying it into start-up zone, medium speed zone, and rated speed zone. Because it does not have a hall sensor, its initial position cannot be identified and thus the motor needs to be aligned in a consistent position for start-up. Because BLDC motor is generally developed according to the six sequences of $120^{\circ}$ two-phase commutation method, it is always aligned to the position if the current is applied by choosing one of the six sequences. Once it is aligned, synchronous acceleration will be applied until the motor reaches a speed in which back EMF can be detected. Since the synchronous acceleration area applies the fixed six sequences to a consistent voltage duty without using hall sensor, it must be observed carefully to prevent step-out due to disturbance from load change. Motor must be driven at a low speed not to have step-out [14-15], and the mode must be changed immediately once the back EMF is detected.

\subsection{Driving method depending on speed zone}

When the ZCP of the existing back EMF is detected, noise occurs during switch on/off as voltage is applied in the pulse form according to PWM control. A sensing error can occurs due to an instant noise in the ZCP detection sensing of back EMP because voltage pulse is detected 


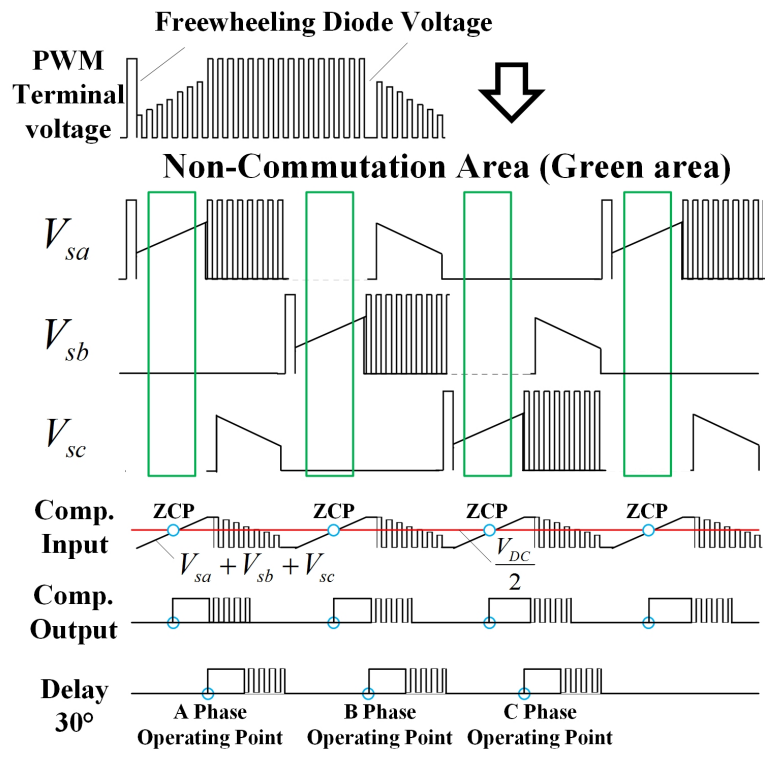

Fig. 7. Sensorless control method including non-commutation area

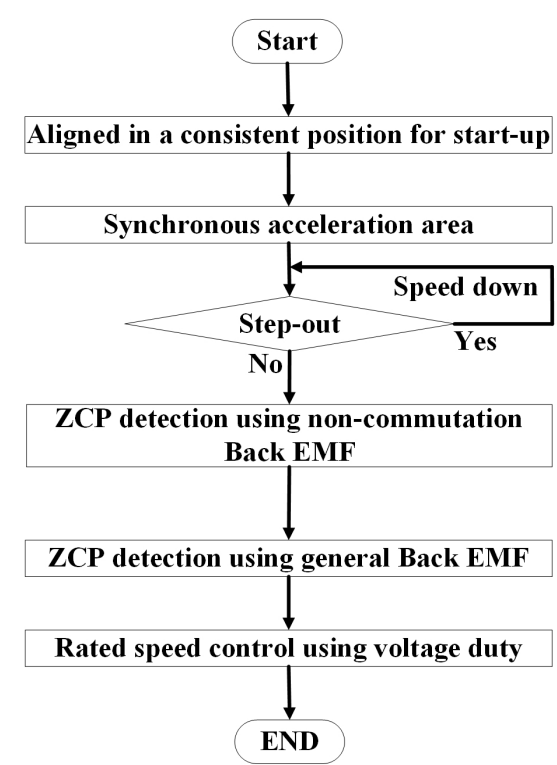

Fig. 8. Sequence diagram of sensorless BLDC motor drive using the proposed $\mathrm{ZCP}$ detection method

immediately once the waveform of terminal voltage is greater than $\mathrm{Vdc} / 2$. Such phenomenon is more severe in the low speed zone that has a smaller size of back EMF, but this problem is solved once the motor reaches the rated speed. Thus, the proposed detection method can be applied for safe switching sensing in the medium speed zone after start-up. Since the motor is a rotating machine, it has inertia when it rotates; as a result it rotates for a while by inertia even when current is not applied by turning the switch off. Since back EMP always appears without applying current when the motor rotates, when the switch is turned off by designating a non-commutation area, only a pure back EMF waveform not included in terminal voltage appears in the area. The control method including the non-commutation area is shown in Fig. 7.

While $120^{\circ}$ two-phase commutation method carries out commutation in order by changing switches for every $60^{\circ}$, the method including non-commutation area carries out commutation and non-commutation in order for every $60^{\circ}$. The motor rotates only by inertia in the non-commutation area. In this area, the motor repeats commutation and noncommutation and thus causes vibration while driving. However, due to the driving nature of ACM, momentary vibration in the driving process is not a huge problem because the motor needs continuous driving only at the rated speed without speed variability. The power of

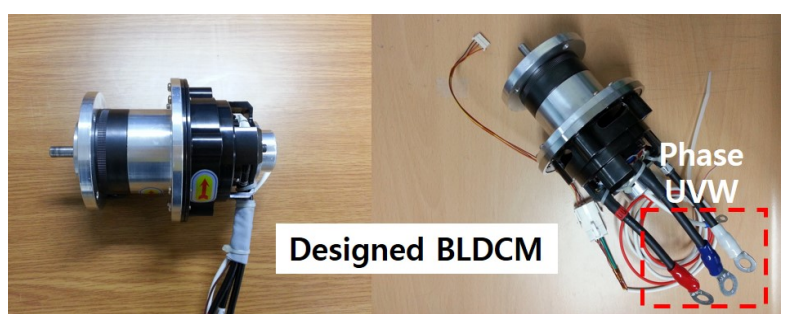

Fig. 9. The manufactured BLDC motor

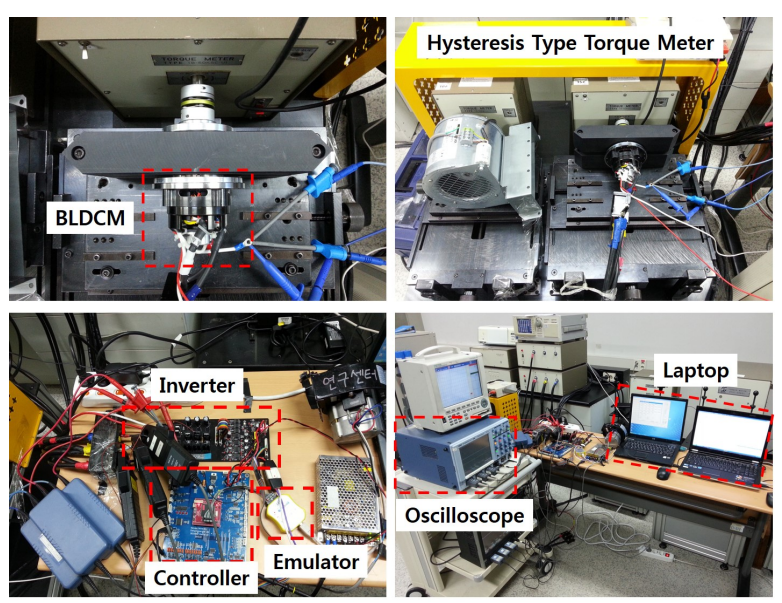

Fig. 10. Motor test system

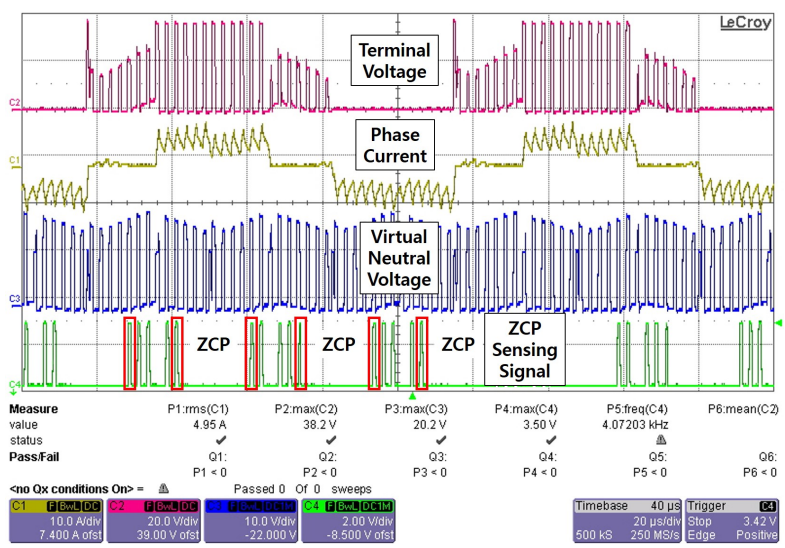

Fig. 11. Experiment waveform depending on $3000 \mathrm{rpm}$ 
comparator is a pulse that includes ZCP and is ahead of the actual commutation position by $30^{\circ}$. Therefore, the motor will be commutated using $30^{\circ}$ phase lag algorithm. Fig. 8 is a sequence diagram of BLDC motor driving process that used the proposed ZCP detection.

\section{Motor Performance Test}

The manufactured BLDC motor is shown in Fig. 9. The motor performance test system is shown in Fig. 10. Using the proposed ZCP detection method, a test was conducted at the rated speed. The motor is controlled using the noncommutation area at the medium speed, and the speed is increased to the rated speed when ZCP starts to be detected normally. The Fig. 11 shows that the motor drives normally until it reaches the rated speed.

\section{Conclusion}

ACM is used for a stable supply of necessary current to a railway vehicle. DC motor for ACM driving needs maintenance due to its mechanical friction, so a study is conducted to replace it with BLDC motor. BLDC motor that uses sensor is vulnerable to heat and cannot rotate stably when an error occurs in the sensor. As a result, it is used on switching sensing by applying back EMF ZCP detection circuit, which is relatively simple. Since the size of back EMF is small at the low speed, there is a high probability of sensing error by the applied voltage noise. Therefore, ZCP can be detected through noiseless back EMF if a non-commutation area is set to be used on commutation.

\section{Acknowledgements}

This work was supported by the Human Resources Program in Energy Technology of the Korea Institute of Energy Technology Evaluation and Planning (KETEP), granted financial resource from the Ministry of Trade, Industry \& Energy, Republic of Korea (No. 20154030200900).

This work was supported by the National Research Foundation of Korea (NRF) grant funded by the Korea government (Ministry of Science, ICT \& Future Planning) (No. NRF-2016R1A2A1A05005392).

\section{References}

[1] N. U. Baeg, S. J. Lee, "Railroad Technology," gbbook, pp. 132, 2004

[2] I. K. Kang, "Electric Railway System Engineering,"
Sungandang, pp. 384, 2002

[3] Haifeng Lu, Lei Zhang, and Wenlong Qu, "A New Torque Control Method for Torque Ripple Minimization of BLDC Motors With Un-Ideal Back EMF," IEEE Trans. Power Electronics, vol. 23, no. 2, pp. 950-958, Mar. 2008.

[4] Dwijasish Das, N. Kumaresan, V. Nayanar, K. Navin Sam, and N. Ammasai Gounden, "Development of BLDC Motor-Based Elevator System Suitable for DC Microgrid," IEEE Trans. Mechatronics, vol. 21, no. 3, pp. 1552-1560, Jun. 2016.

[5] Jose Carlos Gamazo Real, Jaime Gomez Gil, "Sensorless Detection of Position and Speed in Brushless DC Motors using the Derivative of Terminal Phase Voltages Technique with a Simple and Versatile Motor Driver Implementation," KIEE Journal of Electrical Engineering and Technology, vol. 10, no. 4, pp. 1540-1551, Jul. 2015

[6] Nikolay Samoylenko, Qiang Han and Juri Jatskevich, "Dynamic Performance of Brushless DC Motors With Unbalanced Hall Sensors," IEEE Trans. Energy Conversion, vol. 23, no. 3, pp. 752-763, Sep. 2008.

[7] Giacomo Scelba, Giulio De Donato, Mario Pulvirenti, Fabio Giulii Capponi and Giuseppe Scarcella, "HallEffect Sensor Fault Detection, Identification, and Compensation in Brushless DC Drives," IEEE Trans. Industry Applications, vol. 52, no. 2, pp. 1542-1554, Mar. 2016.

[8] G. J. Su and J. W. Mckeever, "Low cost sensorless control of brushless DC motors with improved speed range," IEEE Trans. On Power Electronics, vol. 19, pp. 296-302, 2004

[9] Jianwen Shao, “An Improved Microcontroller-Based Sensorless Brushless DC (BLDC) Motor Drive for Automotive Applications," IEEE Trans. Industry Applications, vol. 42, no. 5, pp. 1216-1221, Sep. 2006.

[10] Dae-Kyong Kim, Kwang-Woon Lee, Byung-Il Kwon, "Torque Ripple Reduction Method in a Sensorless Drive for the BLDC Motor," KIEE international transactions on EMECS, vol. 4-B, no. 4, pp. 196-200, 2004

[11] Kwang-Ryeol Sim, Jeong-Ryeol An, "Sensorless Control Method of the BLDC Motor Without Neutral Point," KIEE Trans. P, vol. 61, no. 3, pp. 111-115, Sep. 2012.

[12] In-gun Kim, Hyun-seok Hong and Ju Lee, “A Study on the Control to Compensate Position Sensor Error of the BLDC Motor in an Auxiliary Air Compressor," KIEE Trans., vol. 64, no. 11, pp. 1639-1644, Nov. 2015.

[13] Quang-Vinh Tran, Tae-Won Chun, Hong-Hee Lee, Heung-Geun Kim, Eui-Cheol Nho, "Control Techniques of Sensorless BLDC Motor Drive for a Vehicle Fuel Pump Application," KIEE Trans. vol. 60, no. 10, pp. 1858-1864, Oct. 2011.

[14] Mourad Masmoudi, Bassem El Badsi and Ahmed 
Masmoudi, "DTC of B4-Inverter-Fed BLDC Motor Drives With Reduced Torque Ripple During Sectorto-Sector Commutations," IEEE Trans. Power Electronics, vol. 29, no. 9, pp. 4855-4865, Sep. 2014.

[15] T. H. Kim and M. Ehasani, "Sensorless control of the BLDC motors from near-zero to high speeds," IEEE trans. On Power Electronics, vol. 19, no. 6, pp. 16351645, 2004.

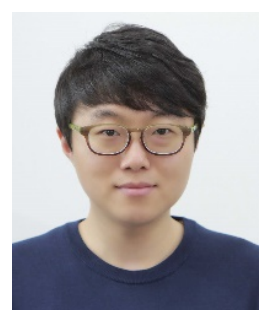

In-Gun Kim He received his B.S. degree in Electrical Engineering from Incheon University, Incheon, Korea in 2011, and his M.S. degree in Electrical Engineering from Hanyang University, Seoul, Korea in 2013. Since 2013, he has been pursuing the Ph.D. degree at the Department of Electrical Engineering, Hanyang University. His research interests include design, testing and control of motor/generator; power conversion systems; and applications of motor drive, such as electric vehicles.

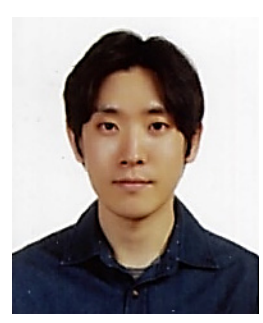

Hyun-Seok Hong He received his B.S. degree in Electrical Engineering from Dankook University, Seoul, Korea in 2012 and M.S. degree in Electrical Engineering from Hanyang University, Seoul, Korea in 2014. Since 2014, he has been pursuing the Ph.D. degree at the Department of Electrical Engineering, Hanyang University. His research interests include design, analysis, testing and control of motor/generator; power conversion systems; and applications of motor drive.

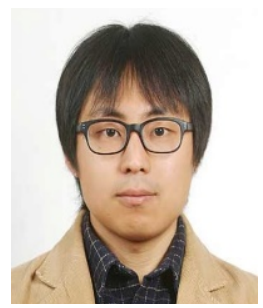

Sung-Chul Go He received his B.S., M.S. and Ph.D. degrees in Electrical Engineering from Hanyang University, Seoul, Korea in 2004, 2006 and 2010, respectively. $\mathrm{He}$ is now working in Samsung Electronics Co. His research interests include design, analysis, testing and control of motor/generator; power conversion systems; and applications of motor drive, such as electric vehicles, high-speed maglev train and renewable energy systems.

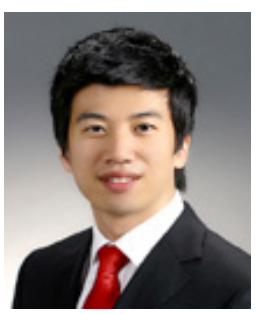

Ye-Jun Oh He received his B.S. degree in Electrical Engineering from Myongji University, Korea in 2010, and his M.S. degree in Electrical Engineering from Hanyang University, Seoul, Korea in 2012. Since 2015, he has been pursuing the Ph.D. degree at the Department of Electrical Engineering, Hanyang University. His research interests include design, analysis and control of motor/generator; power conversion systems; and applications of motor drive, such as electric vehicles.

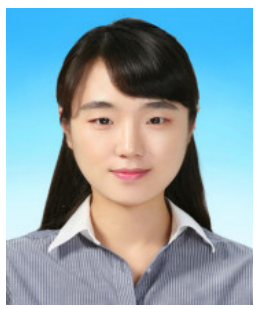

Kyoung-Jin Joo She received her B.S. degree in Electrical Engineering from Changwon National University, Changwon, Korea in 2008, and her M.S. degree in Electrical Engineering from the same university, in 2010. She worked in Korea Electrotechnology Research Institute form 2010 to 2011 and LG electronics from 2011 to 2015. Since 2015, she has been pursuing the Ph.D. degree at the Department of Electrical Engineering, Hanyang University. Her research interests include design, analysis, testing and control of motor/generator; power conversion systems; and applications of motor drive, such as electric vehicles.

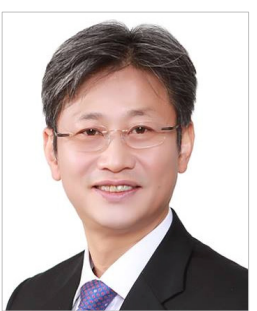

Ju Lee He received his M.S. degree from Hanyang University, Seoul, South Korea, in 1988, and his Ph.D. from Kyusyu University, Japan in 1997, both in Electrical Engineering, $\mathrm{He}$ joined Hanyang University in September, 1997 and is currently a Professor of the Division of Electrical and Biomedical Engineering. His main research interests include electric machinery and its drives, electromagnetic field analysis, new transformation systems such as hybrid electric vehicles (HEV), and high-speed electric trains and standardization. $\mathrm{He}$ is a member of the IEEE Industry Applications Society, Magnetics Society, and Power Electronics Society. 